\title{
Multiboronic acid-conjugated chitosan scaffolds with glucose selectivity to insulin release
}

\author{
Nabil A Siddiqui ${ }^{a}$, Nashiru Billa ${ }^{a *}$ and Clive J Roberts ${ }^{b}$ \\ ${ }^{a}$ School of Pharmacy, The University of Nottingham Malaysia Campus, Semenyih, \\ Malaysia \\ ${ }^{b}$ School of Pharmacy, The University of Nottingham, Nottingham, UK \\ *Corresponding author: nashiru.billa@nottingham.edu.my; Tel.: +6 (03) 89248211; \\ Fax: +6 (03) 89248018
}

\begin{abstract}
The principal challenge for the use of boronic acids (BA) as glucose sensors is their lack of specificity for glucose. We examined the selectivity of and insulin release from two boronic acids- (2-formyl-3-thienylboronic acid (FTBA) and 4-formylphenylboronic acid (FPBA)) conjugated chitosan scaffolds to glucose and fructose. Adsorption of glucose to BA: chitosan conjugates was dose-dependent up to $1: 1$ at 35 and $42 \%$ for FPBA and FTBA respectively but the FTBA conjugates adsorbed more glucose and fructose at respective FPBA ratios. The affinity of both BA conjugates to glucose decreased with increase in BA ratio. On the other hand, the affinity of both BA conjugates for fructose decreased from ratio 1:1 to 2:1 then rose again at 3:1. Insulin release from FPBA nanoparticles (FPBAINP) and FTBA nanoparticles (FTBAINP) were both concentration-dependent within glyceamically relevant values $(1-3 \mathrm{mg} / \mathrm{ml}$ glucose and $0.002 \mathrm{mg} / \mathrm{ml}$ fructose). Furthermore, the total amounts of insulin released from FPBAINP in both the media were higher than from FTBAINP. Both FPBAINP and FTBAINP have the potential for development as a glucose-selective insulin delivery system in physiological settings.
\end{abstract}


Keywords: boronic acid; chitosan; glucose; insulin; nanoparticles

\section{$1 \quad$ Introduction}

As one of the most common chronic diseases in almost every country, the incidence of diabetes is on the rise. Maintenance of continuous normoglycaemic conditions (70-140 mg per $\mathrm{dl}$ or $4-8 \mathrm{mM}$ ) is the key goal for the management of both type 1 and type 2 diabetes [1]. The current treatment option for type 1 and end-stage type 2 diabetes is insulin replacement therapy in the form of multiple daily subcutaneous injections of insulin, which is discomforting and lead to poor patient compliance [2]. This has necessitated research interest amongst scientists working toward developing glucoseregulated insulin replacement therapies that are painless, relatively inexpensive to manufacture compared to the current mode and readily available [3]. In the last two decades, progress has been made in the field of nanotechnology and polymer science whereby scientists have bioengineered nanoparticles to release the pay loaded after sensing changes in their surroundings [4]. Glucose-responsive insulin delivery (GRID) systems are designed to release insulin in response to a rise in glucose levels with a feedback check at normoglycaemic levels. These delivery systems have the potential to not only achieve tighter glycaemic control, but also eliminate the need for frequent finger-stick glucose tests and multiple daily insulin injections [5]. Currently, the three most extensively studied glucose sensors are glucose oxidase, glucose-binding proteins (GBPs) and glucose-binding small molecules. Using these glucose responsive molecules, insulin loaded nanoparticles can be formulated which will release the drug via degradation, disassembly or swelling in response to changes in glucose concentrations $[4,6]$.

Small molecule glucose binders such as boronic acids (BAs) offer a chemical approach to glucose-mediated insulin release via reversible interactions with cis-1,2- or 1,3-diols. 
Unlike glucose oxidase and GBPs, BAs offer the advantages of having fast response rates, being oxygen-independent and causing no immunological responses. However, their lack of glucose specificity has been the major drawback for their use as glucose sensors in GRIDs. It has been shown that monoboronic acids have the highest selectivity for fructose among all the saccharides [7]. This is because the $\beta$-Dfructofuranose (Fig.1a) binds to boronic acids and is available as $25 \%$ of total fructose in $\mathrm{D}_{2} \mathrm{O}$ at $31^{\circ} \mathrm{C}$. On the other hand, the $\alpha$-D-glucofuranose (Fig.1b) form of glucose binds to boronic acids, constituting only $0.14 \%$ of the total glucose in $\mathrm{D}_{2} \mathrm{O}$ at $27^{\circ} \mathrm{C}$ [8]. This poses a constraint for monoboronic acids acting as glucose-specific sensors in systemic insulin delivery systems because blood has both glucose and fructose and insulin release due to the presence of fructose is undesirable in the present context. However, the problem can be overcome by using suitably positioned multiboronic acids anchored on a polymer backbone. Multiboronic acids as glucose-specific sensors are similar to biological sensors like lectins as they have multiple binding sites. Over the last ten years, scientists have proposed several glucose-specific multiboronic acid scaffolds. Currently, four main strategies are being widely investigated which include synthetic diboronic acids, boronic acid-conjugated polymers, self-assembly of simple boronic acids and boronic acid-conjugated nanomaterials [8].

In the present investigation, multiboronic acid scaffolds are formed from a chitosan backbone and studied with regard to their specificity for glucose sensing and subsequent release of insulin in response. In an attempt to address the lack of specificity to glucose sensing by boronic acids, we report for the first time, the use of 2-formyl-3thienylboronic acid (FTBA) as the boronic acid conjugated to chitosan in the development of a glucose chemosensor. Since phenylboronic acids have been widely investigated as glucose sensors, 4-formylphenylboronic acid (FPBA) was chosen for 
comparative studies in this investigation. Equally important is the physical and chemical properties of the scaffold anchoring macromolecule. Chitosan was used as the multiboronic acid anchor in the present study because it is biocompatible, biodegradable, non-toxic and retains a significant level of hydrophilicity relevant for in vivo applications. Furthermore, it is relatively simple and easy to formulate as nanoparticles via polyelectrolyte complexation $[9,10]$. As the nanoparticles form spontaneously in aqueous media with high encapsulation efficiencies, there is no need for the use of additional solvents or heating, hence eliminating concerns of cytotoxicity and insulin instability $[11,12]$. The organic reaction used to conjugate the boronic acids to chitosan is reductive $\mathrm{N}$-alkylation.

\section{$2 \quad$ Materials and methods}

\subsection{Materials}

Low molecular weight chitosan, 2-formyl-3-thienylboronic acid (FTBA), 4formylphenylboronic acid (FPBA), sodium borohydride, glucose hexokinase assay kit and curcumin were purchased from Sigma Aldrich (St. Louis, MO, USA); acetic acid, methanol, acetonitrile, fructose and glucose were purchased from Thermo Fischer Scientific (Bridgewater, NJ, USA); human recombinant zinc insulin from P. pastoris (28.5 IU/mg) was obtained from Merck (Whitehouse, NJ, USA). All other chemicals were of reagent grade.

\subsection{Synthesis and purification of boronic acid-chitosan conjugates}

$50 \mathrm{mg}$ of chitosan (dissolved in $1 \%$ acetic acid) and various molar equivalents of FPBA and FTBA (Table 1) were mixed and stirred in separate clean beakers containing $15 \mathrm{ml}$ of methanol. After 3 hours, sodium borohydride (1.6 x the molar equivalences of FPBA and FTBA) was added in respective beakers and the mixtures were allowed to stir for a further 10 mins. Afterwards, the reaction was quenched with $1 \mathrm{M}$ sodium hydroxide. 
The precipitates formed were centrifuged at $6000 \mathrm{rpm}$ for $10 \mathrm{mins}$ and washed thoroughly with methanol, ethanol and water to remove any traces of unreacted BAs. The conjugates were then lyophilised and stored in $2^{\circ} \mathrm{C}$ for use in further studies.

\subsection{Glucose adsorption studies}

$10 \mathrm{mg}$ of pure chitosan (control) and $10 \mathrm{mg}$ of the various ratios of the conjugates were exposed to $1 \mathrm{ml}$ of $2 \mathrm{mg} / \mathrm{ml}$ glucose solution in Eppendorf ${ }^{\circledR}$ tubes separately. The tubes were placed in an incubator shaker operated at $100 \mathrm{rpm}$ and maintained at $37^{\circ} \mathrm{C}$ for $1 \mathrm{hr}$ of equilibration. Afterwards, the mixtures were centrifuged at 10,000 rpm for 2 mins and the supernatants collected to determine the amount of glucose present via HPLC. The amount present in the supernatant subtracted from the original amount present in the tubes would give the amount of glucose adsorbed by the conjugates.

The HPLC analysis was carried out by first preparing standard solutions of glucose ranging from $0.25-10 \mathrm{mg} / \mathrm{ml} .10 \mu \mathrm{l}$ of each of the glucose solutions was mixed with $1 \mathrm{ml}$ of hexokinase reagent (in excess) in HPLC vials. The mobile phase consisted of 20 $\mathrm{mM}$ potassium dihydrogen phosphate and acetonitrile (95:5). The elution was isocratic with a flow rate of $1 \mathrm{ml} / \mathrm{min}$, whilst the injection volume was $20 \mu \mathrm{l}$ and detection wavelength was $340 \mathrm{~nm}$. The column used was Phenomenex Jupiter ${ }^{\circledR} \mathrm{C}_{18}$ with pore size of $300 \AA$.

\subsection{Boronic acid specificity for diols (glucose and fructose)}

The affinities of the two boronic acids for glucose and fructose were studied using an indirect and novel HPLC technique based on colour changes of curcumin after reaction with boronic acid. The method is based on the premise that curcumin reacts with boronic acids in solution to form a complex called rosocyanine. During this reaction, the colour changes from yellow (curcumin) to bright red (rosocyanine) and both the colours can be determined spectrophotometrically [13]. $10 \mathrm{mg}$ of conjugates (ratios 1:1, 2:1 and 
3:1) were equilibrated with $1 \mathrm{ml}$ of $2 \mathrm{mg} / \mathrm{ml}$ glucose and fructose solutions in Eppendorf ${ }^{\circledR}$ tubes placed in an incubator shaker at $100 \mathrm{rpm}$ and $37^{\circ} \mathrm{C}$. Control tubes had the conjugates exposed to $1 \mathrm{ml}$ of milli-Q water only. After 1 hour, $500 \mu \mathrm{lof} 0.1 \mathrm{mg} / \mathrm{ml}$ curcumin solution (dissolved in methanol) was added in each of the tubes and shaken gently for a few seconds. The following reactions summarise the events that take place after addition of curcumin:

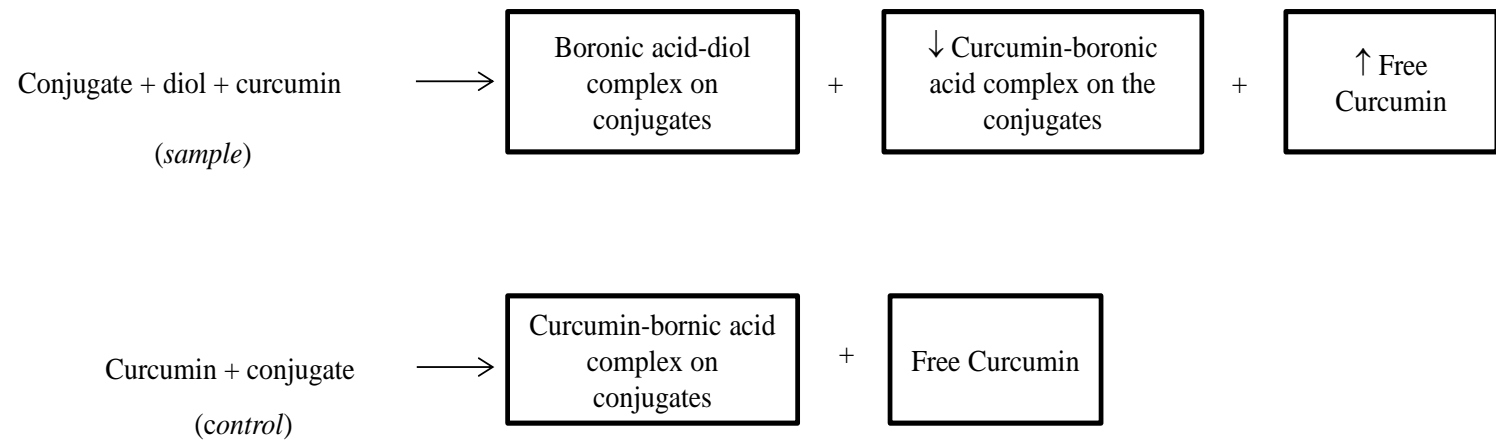

In the control, where curcumin is added after exposure of the conjugates to milli-Q water only, curcumin interacts with all the available boronic acid moieties on the solid conjugates at the bottom of the tube. Since curcumin is present in excess, there is always free curcumin in solution. In the sample tubes, where the conjugates have been equilibrated with glucose or fructose, boronic acid moieties interact with the diols with varying degree of affinities. Consequently, fewer boronic acid moieties are available for interaction with curcumin. This also means that if the same amount of curcumin (as that in control) is added to the sample tubes, the amount of free curcumin in solution will be higher in the sample tubes. Since curcumin can be detected via UV-spectroscopy at 425 $\mathrm{nm}$, the difference in the absorbance between the two respective 'free curcumin' 
solutions will give an indication of the degree of affinity of the conjugates for the two diols. This procedure is sensitive and has not been reported elsewhere.

The HPLC analysis was carried out by first preparing five standard solutions of curcumin ranging from $1-50 \mu \mathrm{g} / \mathrm{ml}$. The mobile phase consisted of acetonitrile, $0.01 \%$ acetic acid and methanol in the ratio 53:42:5. The elution was isocratic with a flow rate of $1.5 \mathrm{ml} / \mathrm{min}$. The injection volume was $10 \mu \mathrm{l}$ and the detection wavelength was 425 nm. The column used was Agilent Zorbax 300SB-4.6 x $250 \mathrm{~mm} \mathrm{C}_{18}$, with particle size of $5 \mu \mathrm{m}$ and pore size of $300 \AA$. To calculate the absorbances of 'free curcumin' with and without the presence of diols, the mixtures were centrifuged at $14,000 \mathrm{rpm}$ for 2 mins and $10 \mu l$ of the supernatants were directly injected into the HPLC system.

\subsection{Formulation of insulin loaded boronic acid-functionalised chitosan}

\section{nanoparticles}

FPBA or FTBA chitosan conjugates (chitosan:boronic acid $=1: 1$ ) was dissolved in $1 \%$ acetic acid to a concentration of $1.5 \mathrm{mg} / \mathrm{ml}$ and the $\mathrm{pH}$ adjusted to $4.2 \mathrm{using} 1 \mathrm{M} \mathrm{NaOH}$. Insulin (dissolved in $0.01 \mathrm{~N} \mathrm{HCl}$ ) was mixed with TPP $(0.5 \mathrm{mg} / \mathrm{ml})$ to give a final concentration of $0.3 \mathrm{mg} / \mathrm{ml}$ and then added dropwise to the conjugate solutions under magnetic stirring to prepare the two nanoparticulate formulations - FPBAINP and FTBAINP.

\subsection{Physical properties of the nanoparticles}

The size (z-average), polydispersity index (pdi) and charge (zeta potential) of the nanoparticles were assessed using a Zetasizer Nano ZS (Malvern, UK) equipped with a $4 \mathrm{~mW}$ He-Ne laser $(633 \mathrm{~nm})$. Each analysis was carried out at $25^{\circ} \mathrm{C}$, performed in triplicate and the data expressed as mean \pm standard deviation. The morphology and surface topography of the nanoparticles was performed on a field emission scanning electron microscope (FESEM, Quanta 400F, FEI Company, Fremont, CA, USA) under 
low vacuum and at a viewing voltage of $20.0 \mathrm{kV}$. After a 1:10 dilution with deionized water, a drop of freshly prepared nanoparticulate solution was placed onto an SEM imaging stub and left to air-dry at room temperature for 24 hours before viewing.

\subsection{In vitro insulin release studies}

The amount of insulin released from the nanoparticles was studied in deionised water, glucose $(1,2$ and $3 \mathrm{mg} / \mathrm{ml})$, fructose $(0.002 \mathrm{mg} / \mathrm{ml})$ and a mixture of glucose $(2 \mathrm{mg} / \mathrm{ml})$ and fructose $(0.002 \mathrm{mg} / \mathrm{ml})$. Several $100 \mu \mathrm{l}$ replicas of FPBAINP and FTBAINP were placed in Eppendorf tubes containing $1 \mathrm{ml}$ of the various media incubated at $37^{\circ} \mathrm{C}$ with horizontal shaking at $100 \mathrm{rpm}$ on a WiseCube WIS-20, Precise Shaking Incubator. At predetermined time points, one of the seeded tubes was withdrawn and centrifuged at $14000 \mathrm{rpm}$ for 5 mins (Beckman Coulter Microfuge 16 centrifuge) followed by direct injection of $20 \mu \mathrm{l}$ of the supernatant onto the HPLC system. The amount of insulin released in the respective media was computed by comparing peak areas obtained with those from the calibration curve.

The HPLC system consisted of an Agilent UV detector (214 nm) linked to an Agilent pump operated by gradient elution: $0.1 \%$ aqueous triflouroacetic acid (TFA) (A) and $0.1 \%$ TFA in acetonitrile (B), with $75 \%$ of $\mathrm{A}$ and decreased to $40 \%$ over 7 min at 1 ml/min using an Agilent Zorbax 300SB-4.6 x 250 mm C 18 column, with particle size of $5 \mu \mathrm{m}$ and pore size of $300 \AA$. The injection volume was $20 \mu \mathrm{l}$. The calibration curve was constructed from pure insulin dissolved in $0.01 \mathrm{~N} \mathrm{HCl}$ at a range of $1-100 \mu \mathrm{g} / \mathrm{ml}$.

\subsection{Evaluation of Encapsulation Efficiency and Loading Capacity of Nanoparticles}

The encapsulation efficiency (EE\%) and loading capacity (LC\%) of insulin within the nanoparticles were determined upon separation of the nanoparticles from the aqueous medium containing free insulin by centrifugation at 14,000 rpm for 45 mins using a Beckman Coulter Microfuge 16 centrifuge (Beckman Coulter, Brea, CA, USA). The 
amount of free insulin in the supernatant was measured using the above HPLC procedure for insulin by comparing peak area obtained with that from the calibration curve. All samples were run in triplicate. The EE\% and LC\% for insulin were calculated as:

$$
\begin{aligned}
& \mathrm{EE} \%=\frac{\text { total insulin in formulation-free insulin in supernatant }}{\text { total insulin in formulation }} \times 100 \\
& (1) \mathrm{LC} \%=\frac{\text { total insulin in formulation- free insulin in supernatant }}{\text { weight of nanoparticles }} \times 100
\end{aligned}
$$

\subsection{Statistical Analysis}

A simple two-tailed t-test was performed with $95 \%$ confidence interval to check for significant differences between experimental results where necessary.

\section{$3 \quad$ Results and Discussion}

\subsection{Glucose adsorption study}

HPLC analyses were carried out to confirm the conjugation of BAs to chitosan through glucose adsorption estimation. Pure chitosan will adsorb very little to no glucose, however, boronic acid conjugated chitosan will adsorb glucose due to the interactions between boronic acid and the hydroxyl moieties of glucose. It is extremely difficult to directly determine glucose via UV-spectroscopy hence glucose hexokinase reagent was used for an indirect estimation. In this reaction, glucose hexokinase phosphorylates glucose to glucose-6-phosphate (G6P) which in turn is oxidised to 6-phosphogluconate (6PG) by glucose-6-phosphate dehydrogenase (G6PD). During the latter reaction, $\mathrm{NAD}^{+}$is reduced to $\mathrm{NADH}$ which can be determined via $\mathrm{UV}$-spectroscopy at $340 \mathrm{~nm}$. Since the amount of NADH produced is directly proportional to the amount of glucose during the entire reaction, the hexokinase assay can give us the amount of glucose adsorbed by the conjugates. 
Fig.2 shows the percentage glucose adsorption at the various ratios of the two boronic acid-chitosan conjugates. Boronic acid is the entity that reversibly binds to glucose, hence pure chitosan adsorbs very little to no glucose. The data clearly shows that the conjugates adsorb glucose.

It can also be seen that, for both the boronic acids, as the ratio of boronic acid to chitosan increases, the glucose adsorption also increases albeit to ratio 1:1. This result is similar to those obtained by Asantewaa et al. [9] who conducted their investigation using FPBA only. It can be hypothesised that at ratios below 1:1, there is insufficient boronic acid moieties within the conjugates to adsorb glucose. Ratio 1:1 appears to provide the optimum number of moieties for maximum adsorption of glucose for the two conjugates. Aptly, at ratios above 1:1, the number of boronic acid moieties on the chitosan backbone increases, however, that also increases the crystallinity of the conjugates [9]. As more and more boronic acids attach to the chitosan backbone, the close packing arrangement makes the structure increasingly rigid. As the two hydroxyl moieties at 1,2- and 3,5,6-positions of $\alpha$-D-glucofuranose (fig.1) are spread out in different domains, a flexible polymer backbone with reasonably spaced boronic acid moieties is required for effective multivalent binding between boronic acids and glucose [8]. This phenomenon might be one of the fundamental reasons as to why the adsorption of glucose decreases above a threshold ratio. Furthermore, the FTBA conjugates (at all ratios) had a higher percentage of glucose adsorption at respective FPBA ratios (significant, $\mathrm{p}<0.025$ ). In FPBA (Fig.3a) the chitosan monomers conjugate with the boronic acids at position 4 of the benzene ring, while in FTBA (Fig.3b), the conjugation takes place at position 2 of the thienyl ring. Due to the proximity of the thienyl rings of FTBA and the cyclohexane rings of chitosan to the glucose molecule as it forms the complex with boronic acid, it can be postulated that additional $\mathrm{C}-\mathrm{H} \bullet \bullet \bullet$ interactions 
might contribute to a slightly higher affinity (and hence greater adsorption) of FTBA to glucose compared to that of FPBA.

\subsection{Specificity of boronic acids for glucose and fructose}

The idea of using curcumin to ascertain the relative affinities of the two boronic acidconjugated polymers for glucose and fructose in this project was envisaged to explore a novel way of studying the extent of adsorption between boronic acids and diols. Curcumin can be used to detect boron at very low (ppm) levels [13], so the slightest change in boronic acid concentrations due to complexation between glucose or fructose can be determined using this dye. A higher amount of free curcumin in solution of an experimental (glucose/fructose) sample compared to that in a control (no diol) sample will indicate the complexation between boronic acids and diols. Furthermore, the difference in free curcumin levels between the diols gives us an estimation of the relative affinities of the boronic acids for glucose and fructose. Fig.4 portrays the results obtained from this investigation. Since glucose adsorption with the conjugate at 1:1 ratio manifested the highest adsorption of glucose (from section 3.1), this conjugate was selected for further investigation. To substantiate our claim that increased crystallinity and/or lack of flexibility within the boronic acid-chitosan scaffolds at higher ratios decreases the sensitivities of the conjugates to glucose adsorption, ratios $2: 1$ and $3: 1$ were also investigated.

It can be seen from Fig.4a that, in the presence of glucose, the amount of free curcumin in solution decreases at conjugate ratios above $1: 1$. This means that at ratios above $1: 1$, more curcumin is involved in complexing with boronic acids. So, it can be deduced that fewer glucose molecules are occupying the available boronic acid binding moieties at higher ratios; in other words, the affinity of the boronic acid-chitosan conjugates for glucose is decreasing. This observation is in concordance with the previous glucose 
adsorption data in section 3.1 and supplements the proposition that lack of flexibility in the polymeric scaffolds at higher boronic acid:chitosan ratios might be the principal reason for the decreased affinity of glucose. Additionally, it should be noted that the $\%$ free curcumin in solution at all FTBA ratios is significantly higher than at corresponding FPBA ratios $(\mathrm{p}<0.025)$. Hence, we may conclude that the FTBA conjugates have greater affinity for glucose than FPBA scaffolds and the notion (presented in section 3.1) that the interactions between the thienyl rings (of FTBA), cyclohexane rings (of chitosan backbone) and the hydrocarbon skeleton of glucose is the reason behind the higher propensity for adsorption of glucose by FTBA appears to be the plausible explanation.

On the other hand, the data with fructose (Fig.4b) contrast those with glucose. Here, the affinity of both types of the boronic acid conjugates for fructose appears to decrease from ratio 1:1 to 2:1. SEM studies conducted by Asantewaa et al. [9] indicate that at ratios above 1:1, the conjugates become less porous and more crystalline with flat surfaces. Consequently, with increase in crystallinity, the surface area:volume ratio decreases which impedes effective interaction between fructose and boronic acids. However, the affinity of boronic acid conjugates for fructose rises again at 3:1 which is a contradiction to the theory put forward by Asantewaa et al [9]. This takes us back to the topic of monovalent interactions of fructose with boronic acid (section 2.1). Wu et al. [8] identified that fructose acts as a monovalent ligand (Fig.3c) in almost all sensing studies performed with boronic acids.

Unlike glucose, which has two diol groups involved in multivalent binding with two boronic acid moieties and thus requiring a flexible polymer backbone, fructose appears to overcome the challenge imposed by increased crystallinity with its monovalent interactions capability. One molecule of fructose can bind with one boronic acid moiety, 
so at higher conjugate ratios where the number of boronic acids attached to chitosan is higher, more fructose molecules come together and attach to boronic acids side by side. Additionally, at ratio 1:1, the specificity of both FPBA- and FTBA-chitosan scaffolds for glucose is highest $(7.58 \%$ and $20.73 \%$ higher level of free curcumin in solution respectively). It can be posited that this is the optimum ratio where the conjugates have the ideal number of boronic acids for effective multivalent interactions with glucose. Multivalency, in chemical and in biological systems, is a key concept which means that when two multivalent moieties are involved in $n$ binding events $(n>1)$, the binding occurs with higher affinity than the sum of $n$ individual monovalent bindings. According to fig.1, fructose has one binding moiety while, glucose has two (at the 1,2and 3,5,6-positions). Consequently, monoboronic acids with one binding moiety interact with greater affinity with fructose since monovalent interactions take place here. However, in di- or multiboronic acids, there are at least two boronic acid moieties available for interaction with one glucose molecule, and hence this interaction is substantially stronger than monovalent interactions which take place with fructose [8]. We should also note that both the glucose and fructose concentrations in this investigation were kept the same $(2 \mathrm{mg} / \mathrm{ml})$. While this is a realistic glucose concentration in biological settings, the fructose concentration in the human blood is a thousandth less $[14,15]$. Considering the fact that the ultimate aim of this project is to develop a GRID for diabetic patients, it can be asserted that both types of boronic acidchitosan conjugates, designed in this study, are glucose selective at physiologically relevant glucose concentration.

\subsection{Insulin-loaded chitosan-TPP nanoparticles}

As boronic acid:chitosan ratio of $1: 1$ has proved to induce the highest glucose adsorption and possess the highest glucose specificity in the previous sections, this ratio 
of conjugate was chosen to prepare the chitosan-TPP nanoparticles. Table 2 shows the size, charge and pdi of the two types of nanoparticle formulations. Overall, the average diameters of the target nanoparticles - FPBAINP and FTBAINP - are reasonably small and the formulations are homogenous (as indicated by the pdi value). The small size of the nanoparticles is relevant to this investigation as it ensures a large surface area to volume ratio, which in turn should improve sensitivity. Nanoparticles with a surface charge of $|10| \mathrm{mV}$ are considered approximately neutral; while nanoparticles with zeta potentials of at least $|30| \mathrm{mV}$ are considered strongly ionic, thereby ensuring sufficient repulsive forces to keep the particles apart [16]. In that respect, the overall charges of the formulations are slightly on the lower side. Notwithstanding, FPBAINP and FTBAINP appear to be reasonably separated from each other as shown in the FESEM images in Fig.5. The particles also appear spherical and in agreement with the z-average determinations.

\subsection{Entrapment of Insulin within Chitosan Nanoparticles}

The drug release profile and pharmaceutical cost-effectiveness of a formulation depend on the drug loading capacity or the encapsulation efficiency (EE \%) within the carrier system. This is particularly crucial for nanoparticulate delivery systems which have the smallest surface area-to-volume ratio of all dosage forms. The EE\% for FPBAINP and FTBAINP were $56.7 \%$ and $57.5 \%$ respectively. There is a range of reported EE\% of insulin in nanoparticulate drug delivery systems. Zhang et al. [17] reported an insulin loading capacity of more than $78 \%$ in their polyethylene grafted chitosan nanoparticles, whilst Zhu et al. [18] prepared PEG modified N-trimethylaminoethylmethacrylate chitosan nanoparticles which resulted in a range of $\mathrm{EE} \%$ from $10-84 \%$ depending on the initial weight of the polymer used in the formulation. Wu et al. [19] formulated nanoparticles with an EE\% ranging from $49-59 \%$ and contend that this variability was 
attributed to the amount of insulin used and the molecular weight of the polymer. We may conclude that the EE\% obtained in the present investigation is at least comparable to those reported in the literature. The LC\% for FPBAINP and FTBAINP were calculated to be $45 \pm 1.4 \mathrm{mg}$ and $48 \pm 1.1 \mathrm{mg}$ of insulin in $100 \mathrm{mg}$ of nanoparticles respectively.

\subsection{In vitro insulin release in various media}

In section 3.2, we have discussed the superior specificity of FPBA- and FTBA-chitosan conjugates (at BA:chitosan ratio of $1: 1$ ) for glucose compared to fructose through a review of the chemistry of the conjugates in the presence of the diols. The current section is aimed at substantiating the selectivity of the two conjugates for glucose via the physiochemical properties of the nanoparticulate formulations of the scaffolds. Three representative blood glucose concentrations were used to study the glucose dependency for insulin release; deionised water was used to investigate the natural diffusion of insulin from the nanoparticles; representative blood fructose concentration (diabetic) was used to investigate the influence of fructose on insulin release and a mixture of glucose and fructose was used to confirm glucose specificity of the boronic acid chitosan scaffolds. Fig.6a and $\mathrm{b}$ show the insulin release profiles of the two nanoparticle formulations in the six media investigated. In FPBAINP (Fig.6a), an initial burst phase of insulin release followed by a more sustained release was observed in all media. The burst phase can be attributed to the release of insulin that is entrapped toward the edge of the nanoparticulate matrix. The sustained release is of the insulin that is present within the deeper pockets of the core matrix. However, the amount of insulin released during the burst phase varied depending on the external media.

A similar pattern of insulin release was observed in FTBAINP formulation as well. In both FPBAINP and FTBAINP, the amount of insulin released within the first 10-20 
mins increased as the concentration of external glucose media increased from $1-3$ $\mathrm{mg} / \mathrm{ml}$. In fact, within the first 20 mins the amount of insulin released from both the formulations in glucose media $(3 \mathrm{mg} / \mathrm{ml})$ was at least twice that in glucose media (1 $\mathrm{mg} / \mathrm{ml}$ ). Springsteen et al. [20] noted that at higher glucose concentrations, the rate of interaction between glucose and boronic acid is higher. We believe that insulin release is preceded by swelling of the scaffolds caused by an increased level of interactions between boronic acid and glucose. This confirms the glucose concentration-dependent insulin release potential of the nanoparticles.

The total amount of insulin released after one hour is identical in both FPBAINP and FTBAINP. Both formulations have minimal leakage as the total amount of insulin released in deionised water after one hour is below $4 \%$. Interestingly, the total amount of insulin released from FPBAINP in fructose is higher than that released from FTBAINP in the same media. This observation might be the result of a relatively limited number of interactions between fructose and FTBA moieties than with FPBA moieties and hence allude to the fact that FTBA has lower affinity for fructose than FPBA. This observation was also noted in the raw conjugates and has significant implications in developing a GRID. It should also be noted that the amount of insulin released from both the formulations in fructose (of blood concentration) is similar to that released in deionised water and/or glucose $(1 \mathrm{mg} / \mathrm{ml})$. Furthermore, in glucose + fructose media, the total amount of insulin released from both the systems is similar to that released in $2 \mathrm{mg} / \mathrm{ml}$ glucose media. Considering the fact that both media had the same concentration of glucose and the only difference was the (blood concentration) of fructose, it can be concluded that both FPBAINP and FTBAINP have the potential for glucose selectivity in physiological settings. The presence of physiological 
concentrations of fructose does not appear to have a marked effect on insulin release from the two systems.

\section{Conclusions}

Both boronic acid-chitosan conjugates and nanoparticles were sensitive to glucose in biologically relevant settings, with 2-formyl-3-thienyl boronic acid possessing slightly higher affinity for glucose. Boronic acid-chitosan ratio of $1: 1$ proved to possess the highest glucose selectivity and was be used to conduct insulin nanoparticle formulation and release studies. Both systems released insulin predominantly in the presence of glucose. We have shown separately that the release of glucose is preceded by expansion of the nanoparticles arising from primary interactions between boronic acid moiety and diols [10]. We may conclude that the presented systems have significant potential for development and application as GRID systems.

\section{Conflicts of interest}

The authors declare that they have no competing interests.

\section{Acknowledgments}

The authors gratefully acknowledge the support for this study by the University of Nottingham. 


\section{FIGURES}

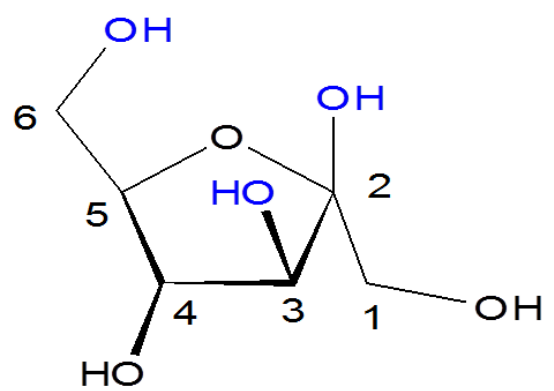

(a)

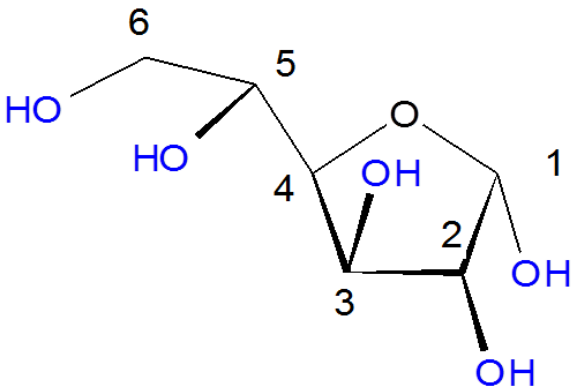

(b)

Figure 1: $\quad$ Potential boronic acid binding sites with (a) fructose and (b) glucose.

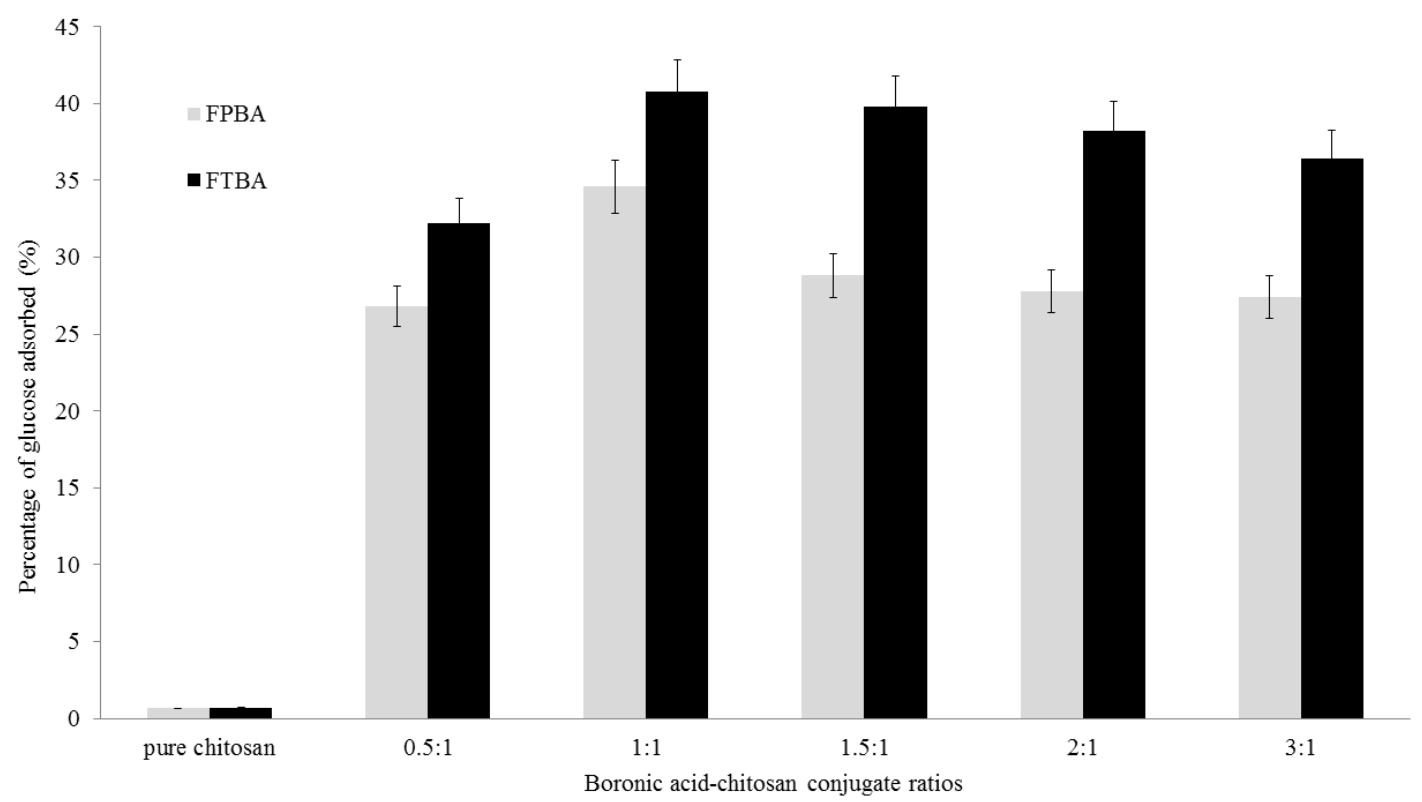

Figure 2: $\quad$ Glucose adsorption of FPBA and FTBA conjugates 


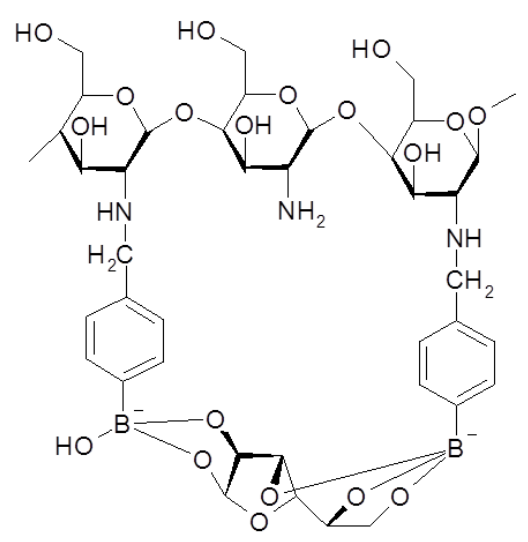

(a)

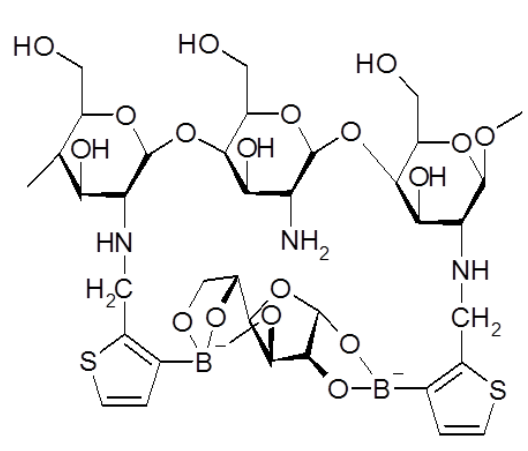

(b)

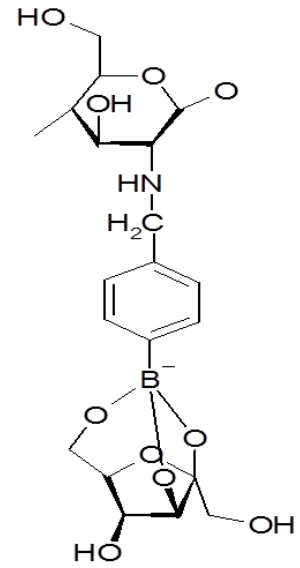

(c)

Figure 3: $\quad$ Structures of $\alpha$-glucofuranose complex with (a) FPBA-chitosan and (b) FTBA-chitosan conjugates and (c) $\beta$-fructofuranose with FPBAchitosan.

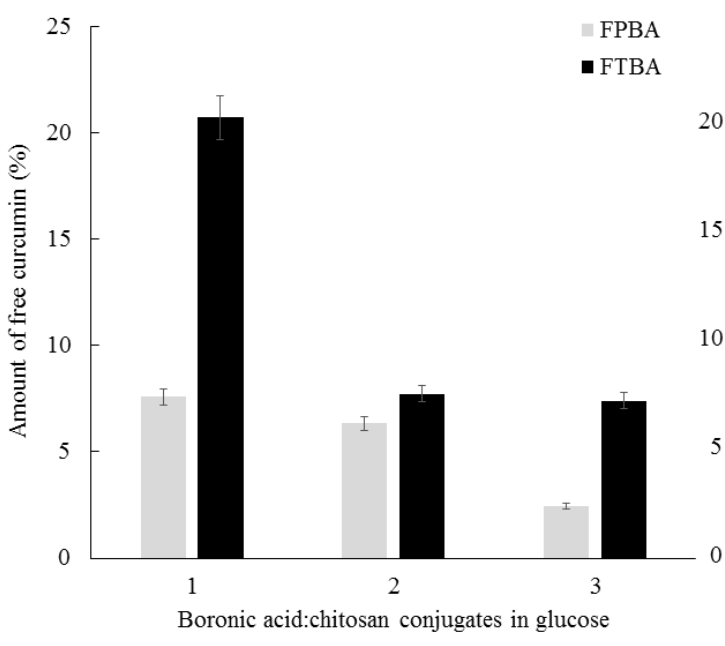

(a)

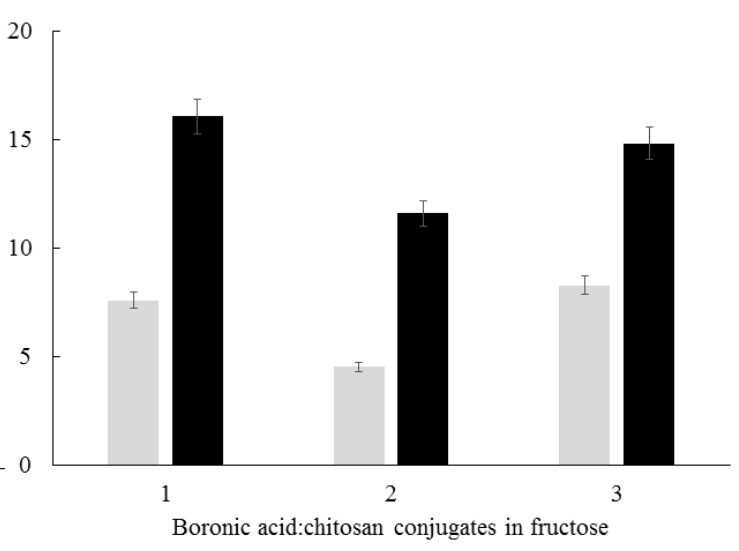

(b)

Figure 4: $\quad$ Selectivity of FPBA- and FTBA-chitosan conjugates in (a) glucose and (b) fructose media in terms of amount of free curcumin (\%). 


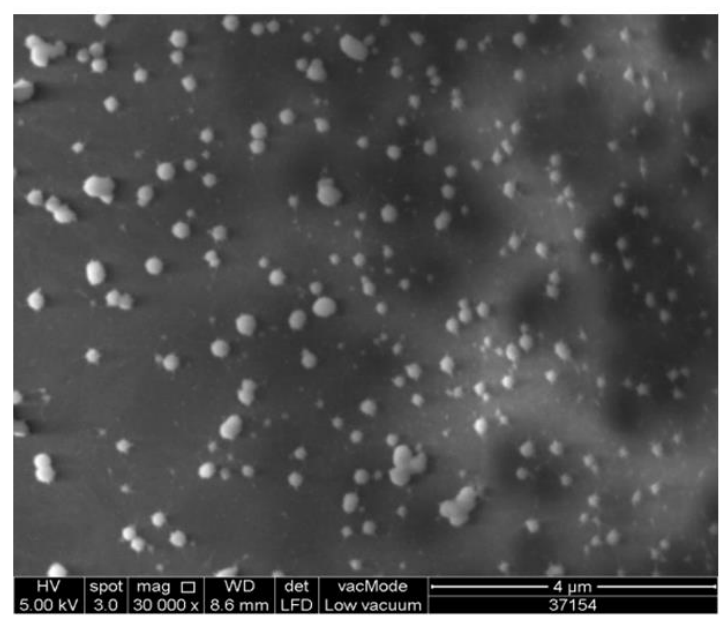

(a)

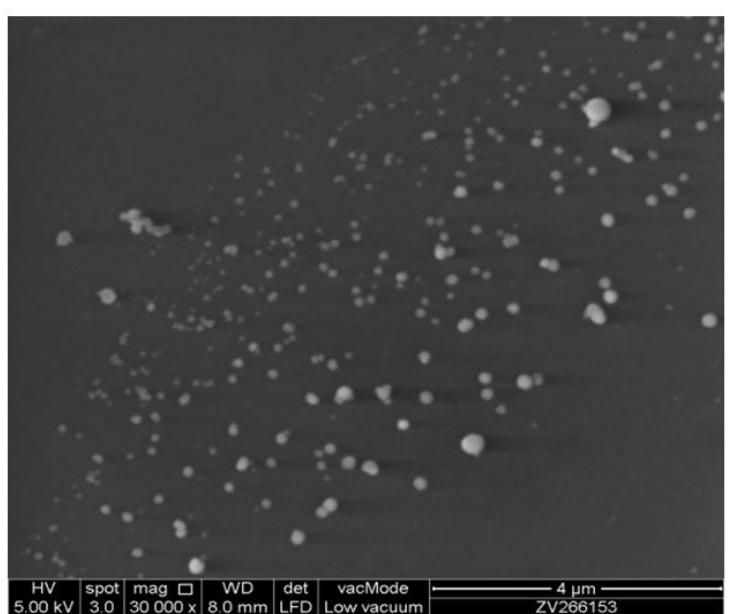

(b)

Figure 5: $\quad$ Scanning electron microphotography of (a) FPBAINP and (b) FTBAINP
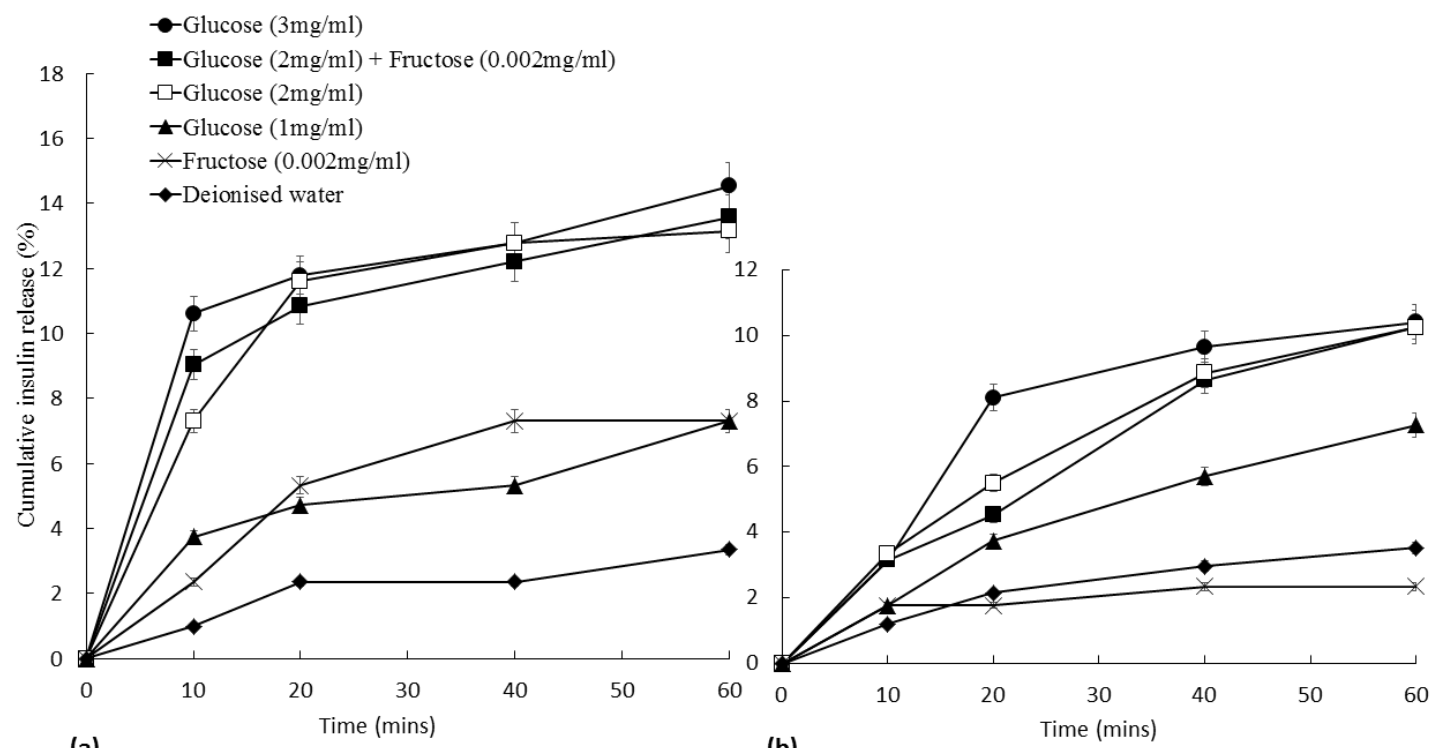

(b)

Figure 6: $\quad$ Insulin release profiles of (a) FPBAINP and (b) FTBAINP in various media. 


\section{References}

1. American Diabetes Association. Standards of medical care in diabetes. Diabetes Care. 2013. 36, 11-66.

2. Tamborlane W. Continuous glucose monitoring and intensive treatment of type 1 diabetes. N. Engl. J. Med. 2008; 359, 1464-1476.

3. Pickup J. Insulin-pump therapy for type 1 diabetes mellitus. N. Engl. J. Med. 2012; 366, 1616-1624.

4. Stuart M, Huck W, Genzer J, et al. Emerging applications of stimuli-responsive polymer materials. Nature Mater. 2010; 9, 101-113.

5. Zion T. Glucose-responsive materials for self-regulated insulin delivery [Thesis]. Massachusetts (MA): Massachusetts Institute of Technology; 2004.

6. Wu W, Mitra N, Yan E, et al. Multifunctional hybrid nanogel for integration of optical glucose sensing and self-regulated insulin release at physiological $\mathrm{pH}$. ACS Nano. 2010; 4, 4831-4839.

7. Lorand J, Edwards J. Polyol Complexes and Structure of the Benzeneboronate Ion. J. Org. Chem. 1959; 24, 769-774.

8. Wu X, Li Z, Chen $X$, et al. Selective sensing of saccharides using simple boronic acids and their aggregates. Chem. Soc. Rev. 2013; 42, 8032-8048.

9. Asantewaa, Y.; Aylott, J.; Burley, J.; Billa, N.; Roberts, C., 2013. Correlating physicochemical properties of boronic acid - chitosan conjugates to glucose adsorption sensitivity. Pharmaceutics. 5, 69-80.

10. Siddiqui NA, Billa N, Roberts CJ, et al. Cross-Linked Dependency of Boronic Acid-Conjugated Chitosan Nanoparticles by Diols for Sustained Insulin Release. Pharmaceutics. 2016; 8, 30. 
11. Makhlof A, Tozuka Y, Takeuchi H. Design and evaluation of novel $\mathrm{pH}$-sensitive chitosan nanoparticles for oral insulin delivery. Eur. J. Pharm. Sci. 2011; 42, $445-51$.

12. Sonaje K, Chuang E, Lin K, et al. Opening of epithelial tight junctions and enhancement of paracellular permeation by chitosan: microscopic, ultrastructural, and computed-tomographic observations. Mol. Pharm. 2012; 9, 1271-9.

13. Lawrence K, Flower S, Kociok-Kohn G, et al. A simple and effective colorimetric technique for the detection of boronic acids and their derivatives. Anal. Methods. 2012; 4, 2215-2217.

14. Kawasaki T, Akanuma H, Yamanouchi T. Increased fructose concentrations in blood and urine in patients with diabetes. Diabetes Care. 2002; 25, 353-357.

15. Yao Y, Zhao L, Yang, J. Glucose-responsive vehicles containing phenylborate ester for controlled insulin release at neutral pH. Biomacromolecules. 2012; 13, $1837-44$.

16. Clogston JD, Patri AK. Zeta potential measurement In Characterization of Nanoparticles Intended for Drug Delivery. New York (NY): Humana Press; 2011.

17. Zhang X, Zhang H, Wu Z, et al. Nasal absorption enhancement of insulin using PEG-grafted chitosan nanoparticles. Eur. J. Pharm. Biopharm. 2008; 68, 526534.

18. Zhu S, Qian F, Zhang Y, et al. Synthesis and characterization of PEG modified N-trimethylaminoethylmethacrylate chitosan nanoparticles. Eur. Polym. J. 2007; 43, 2244-2253. 
19. Wu Z, Zhang S, Zhang $\mathrm{X}$, et al. Phenylboronic acid grafted chitosan as a glucose-sensitive vehicle for controlled insulin release. J. Pharm. Sci. 2011; 100, $2278-2286$.

20. Springsteen G, Wang B. A detailed examination of boronic acid-diol complexation. Tetrahedron. 2002; 58, 5291-5300. 